\title{
Real-time Location Based Shared Smart Parking System
}

\author{
Pragati Kanchan ${ }^{1, *}$, Chhaya Mhaske ${ }^{1}$, Reena Pagare ${ }^{1}$, and NikhilKumar B. Shardoor ${ }^{l}$ \\ ${ }^{1}$ Department of CSE, School of Engineering, MIT ADT University, Pune, India
}

\begin{abstract}
With the growth in population, traffic congestion and parking have become a serious problem. There is the explosive growth of the per capita amount of vehicles. In this paper, a Location-Based Shared Smart Parking System is proposed to solve the parking problem. This system is designed for both private and public parking areas. This system helps the user to find the nearest possible parking area and gives the information related to the availability of parking slots in that respective parking area. The main focus of this system is to reduce the time for finding the parking slot and also avoids unnecessary travelling through occupied parking slots in a parking area. The proposed system is designed using loT technology, WSN(IR sensors), QR code and RFID technology. IR sensors are used to detect the presence of the car in the parking slot and QR code is used to authenticate the user in the public parking area, RFID technology is used to authenticate the user in private parking area. The system is expected to reduce traffic problems, fuel consumption which results in reduces carbon footprints in an atmosphere.
\end{abstract}

\section{Introduction}

With the increase in Indian population, and change in people's lifestyle, every family own a vehicle today. As India's population increases rapidly this has led to rapid increase in number of vehicle on the road. Also inconvenience in public transportation is one of the major reason for increase number of vehicle on road. Parking space in Indian cities has remained constant or reduce due to a growing population. In the current situation, there is no standard system for finding parking space in parking area. Existing systems are timeconsuming and depends on human interaction. During festive season people visit the mall and after reaching to the parking area they face the problem related to limited parking area. Thus, there is a requirement for a shared smart parking system. This will help the user to find a suitable location for parking.

People who are searching for parking location causes traffic congestion. Mostly this issue is occurring in city areas. F. I. Shaikh et al. [11], researchers have found that in one year, car cruising for parking burning 1.7 lakh litre of fuel and producing 730 tons of $\mathrm{CO} 2$, which increases air pollution. Smart Parking systems generally collects the information related to available parking slots in a particular geographic area and the process is real-time to help the user to place vehicles at available positions also, make use of shared parking resources. Shared parking help to optimize parking space in urban areas. Where private parking owners such as hotels, companies, hospitals, etc. and one can rent out their parking space at times they don't use it. In this paper, a real-time location based shared smart parking system based on IoT technology is proposed which enable the user to find the nearest suitable parking space based on their location via mobile application. QR code and RFID technology are used to increase safety and security.

Nowadays, searching for a parking slot in city areas becomes a serious issue. Most of the time people are wasting their time searching for a parking slot. Drivers keep searching for parking space leads to an increase in traffic flow. Searching for empty parking slot during peak hours not only time-consuming but also result in wastage of fuel. Searching for parking burn excess amounts of fuel-producing $\mathrm{CO} 2$ results in increased air pollution. Hence shared smart parking becomes a need of the day.

\section{Related Work}

T. N. Pham et al. [1] in this paper introduces a novel algorithm for the current cloud-based smart parking system to increase efficiency and develop a network architecture based on IoT technology. This system helps the user to find free parking space with the least cost. In this paper, authors have proposed and implemented a smart parking system based on IoT technology. This system uses WSN with RFID technology for monitoring car parks. In this paper system is design by considering only distance parameter from user location to parking location but percentage of available parking space is not consider.

S. R. Basavaraju et al. [2] in his work proposed a smart parking system. which helps the user to search the

\footnotetext{
*Corresponding author: pragatikanchan04@gmail.com
} 
nearest parking area and availability of parking space. The aim of this system is to reduce the time for searching parking space which, leads to reduce fuel consumption and also reduces air pollution. This proposed system is based on IoT Technology which, is consists of a Raspberry Pi-based parking system and $\mathrm{Pi}$ camera. It uses a centralized server to maintain databased which having information about parking space available in the city.

B. M. K. Gandhi et al. [3] this study proposed a parking management system that helps a user to find the availability of parking space using IoT technology. This system consists of IR Sensors, which are placed at the centre of each parking slot to detect a car. Users can access or check the availability of slots and prices remotely using this system. Where all data is stored in the cloud at the centralized system. RFID tags technology is also used in this system to detect the car details incase of identifying or detecting car theft.

C. Shiyao et al. [4] in this study, the system is built using the ZigBee network to send urgent information to the PC through a coordinator and for making updation in databases. This paper presents design and implementation of an Smart parking system.

J. Xiao et al. [5] in this paper Markov $\mathrm{M} \backslash \mathrm{M} \backslash \mathrm{C} \backslash \mathrm{C}$ queue model and stochastic queuing model is used. This model are used to predict the future occupancy from previous occupancy data. This paper are used to estimation of model parameter and occupancy prediction parameter. Accuracy attribute is used in this system.

W. Alajali et al. [6] in his work GBRT and k-cross validation technology for car parking prediction in smart city is used. In this paper three dataset are used like a street car parking dataset, pedestrian data and car traffic data. Here accuracy attributes is used.

E. Simhon et al. [7] in this study RMSE (minimize root mean square error) is used. These prediction occupancy rate of all neighbour area and target occupancy rate. The prediction is based on level of occupancy rate of past data. This method is cost efficient for parking space. The main advantage of this paper is through mobile app find the parking slot.

Y. Zheng et al. [8] in his work the three algorithm like a regression tree, SVM and $\mathrm{NN}$ are used for real time prediction of car parking occupancy rate. The parameter are used like public safety, transport logistics and environment management. Here dataset are used for data collection of cities. This system are based on time interval.

S. Yang et al. [9] in this paper GCNN, RNN and LSTM technology are used for prediction of occupancy of parking. This system uses input parameter like a parking meter transaction, traffic speed and weather condition. The accuracy attribute are used by using mean absolute error formula.
J. Hu et al. [10] in his work introduces the block-chain based parking system for managing parking services and securely storing user information. The proposed system is based on the BlockChainOpenSource(BCOS) framework and smart contract technology for shared space. This system uses a consortium blockchain derived from ethereum technology. A smart contract is used for registration and transaction module for both the parking space owner and users. It allows users to search for a vacant parking space. The aim of this system is to reduced system maintenance and recovery cost by omitting a centralize party to control the entire system. This system is designed for paid parking resources. The vehicle user needs to pay the amount after leaves the parking lot to the owner's payment address through BCOS cash. It also provides a one-time pad mechanism and a group signature mechanism.

The study shows that there is a need to optimize management and labour costs for optimum use of resources for car-park owners. There is also less work found which covers the security aspect during parking. The time utilization can also be optimized by reducing the average waiting time of users for searching parking space. This will result in reduced air pollution and traffic congestion.

\section{Problem Statement}

A shared smart parking system is help the user to find the most suitable and nearest parking space in real-time based on their location. It enables the user to make online reservation of parking slot also allow parking administration to define and manage parking area.

\section{Proposed System Architecture}

The real-time location based share smart parking is designed for both the public and private shared parking.

\subsection{Scenario I}

This system is based on IoT technology. The system uses WSN(Infrared Sensors). Infrared sensors placed in parking slots used to identify the presence of a car at the parking slot in public parking area. It gives information about the parking slot is empty or not. After login into the system, the user will be able to access location-based information and make a reservation for parking slot through the mobile application. The user makes the request for an available parking slot. Information related to the parking slot gather by IR sensors is send to microcontroller and later stored on the centralized system i.e. on a cloud through web services. Shared smart parking system check the availability of parking slot based on information stored on a cloud. If the parking slot found empty it allocates that slot to the user who makes the reservation for that slot and sends QR code to user in response as shown in Fig. 1. The QR code technology is used to identify and authenticate the user against their parking reservations. After getting $\mathrm{QR}$ code user makes use of that code for making entry into 
parking. Whenever a new car enters into parking area system will updates parking space status in real-time. The Google Map API use the users geolocation and location of the parking spots to determine the nearest available parking[11].

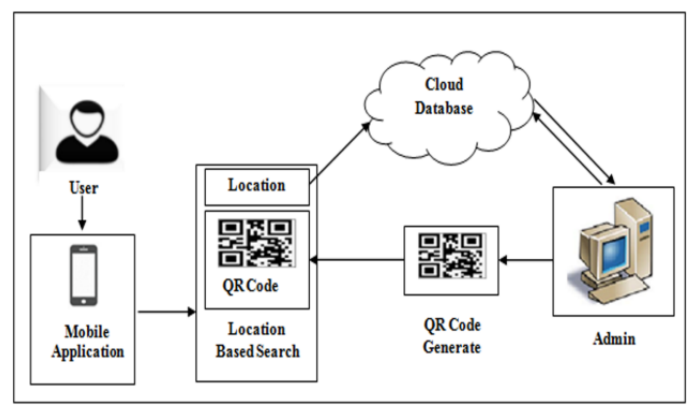

Fig. 1. Shared Smart Parking System.

\subsection{SYSTEM FLOWCHART}

Whenever the user wants to search for a parking, user $\log$ into the system. After, login user request for parking slot based on their location or given area. Then, the user's request is sent to the system, it checks the availability of given slots into the database. If a parking slot is available that slot is reserved for that user and then system generates a QR code and sends it to the user in response. If the parking slot is not available it sends a message to the user that no parking slot available. If a parking slot is available and the user gets acknowledgment. At the time user enters into parking QR code is verify and allow the user to park their vehicle. Otherwise, the user will not get entry into parking, as shown in Fig. 2.

\subsection{Scenario II}

Private or localized share smart parking system enables users to use the parking area of hotels, hospitals, commercial buildings, etc. at a time when there is a free parking slot available. This can be done by taking permission from owners of the parking area. Suppose, During festive season person visit mall and after reaching to parking area he experience that there is no slot is available for parking. In that situation, helshe can make uses of the private parking slot with the help of shared smart parking system. A real-time location-based shared smart parking system for private parking area is consists of IoT technology, Infrared Sensors, and RFID technology. The system is placed on the private parking area which continuously monitoring empty parking slots with the help of IR sensors and the same information is get reflected into the cloud sever. IR sensors are placed in the parking area which will continuously check the availability of slots in parking and send information to the microcontroller. Information gathered by an Infra-red sensor is later stored on the cloud database. If there is a vacant parking slot then Green LED is blown and if the parking slot is reserved then the Red LED is blown. The RFID technology is used for authentication of the user so that only authorized persons can make entry in the parking. To use this system user first need to register through the mobile application. Information filled by the user(like Id, Name, Vehicle number) is stored on a cloud database and RFID Tag.

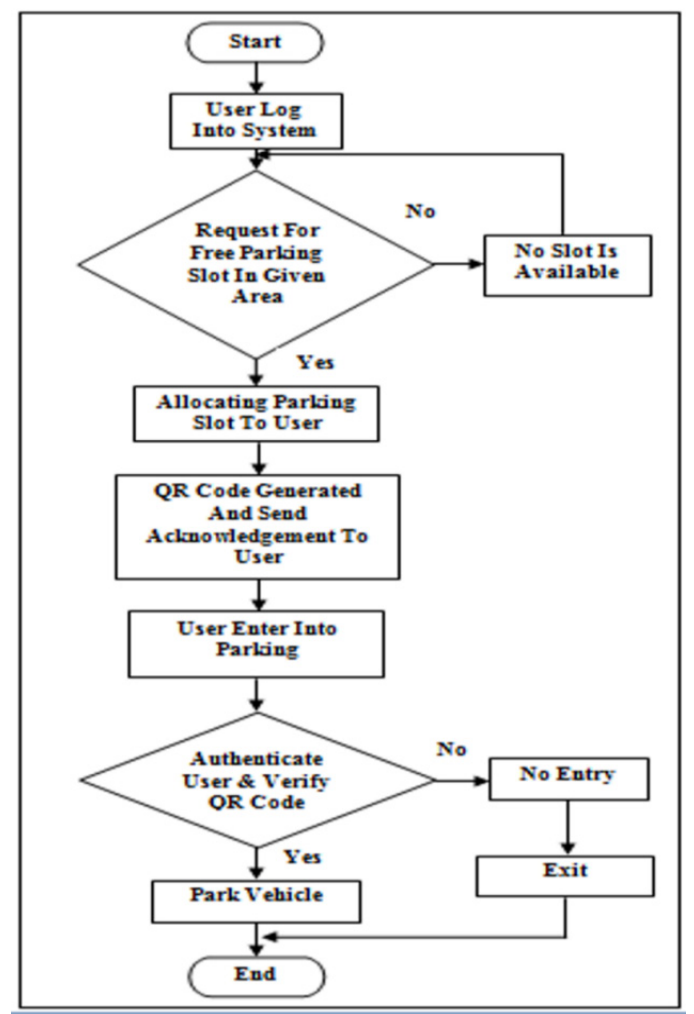

Fig. 2. Flowchart of Shared Smart Parking System

RFID Tag Id is given to registered users for identification and authentication purpose.

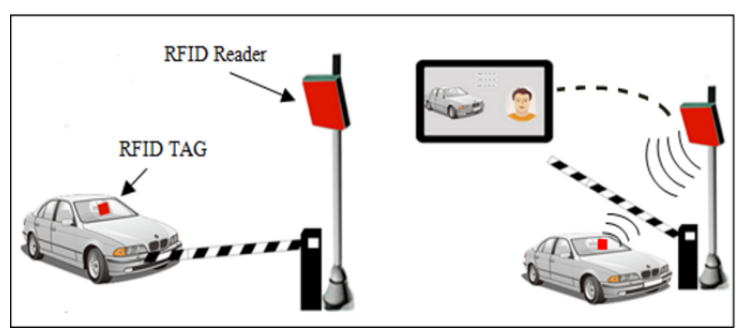

Fig. 3. Parking System with RFID Technology for private uses.

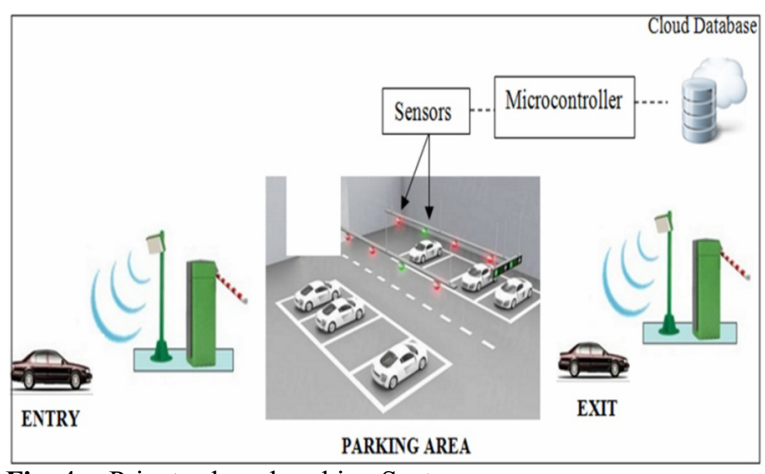

Fig. 4. Private shared parking System 
The tags having electronically stored information. There are two types of RFID tag, Active Tag and Passive Tag. Passive Tags take energy from the nearest RFID Reader's interrogative radiowaves[12]. User first logs into the system, after login user can search for parking location based on their choice. Based on location entered by the user, the application will show related parking areas and availability of slots in that particular location. The user makes the reservation for an available slot through the mobile app. RFID Tag is placed on the car, when the user reached to that parking area at entry gate, RFID Reader reads the data from that tag through radiowaves, that data is checked with the cloud database. If user data is matched then allow the user to make entry into the parking otherwise if the information is not matched or if there is no RFID Tag on the car then that user will not get entry into the parking area. Similarly, when the user leaves a parking area user data is scan and status is updated to the database as shown in above figure Fig. 3 and Fig. 4. The vehicle user needs to pay the amount after leaving the parking slot to the owner's payment address.

\section{Results And Discussion}

Comparison of two scenarios shown in Fig. 5. and Fig. 6.

1) Parking System without Shared Smart Parking

2) Parking System with Shared smart Parking

With an existing parking system, people are wasting their time finding parking space, searching for parking space is not only increased traffic on road but also burn excessive amounts of fuel, producing $\mathrm{CO} 2$ results in increased air pollution. As shown in Fig. 5., the given graph depicts that if searching time increase, the traffic will also increase, which results in an increasing carbon footprint in the environment.

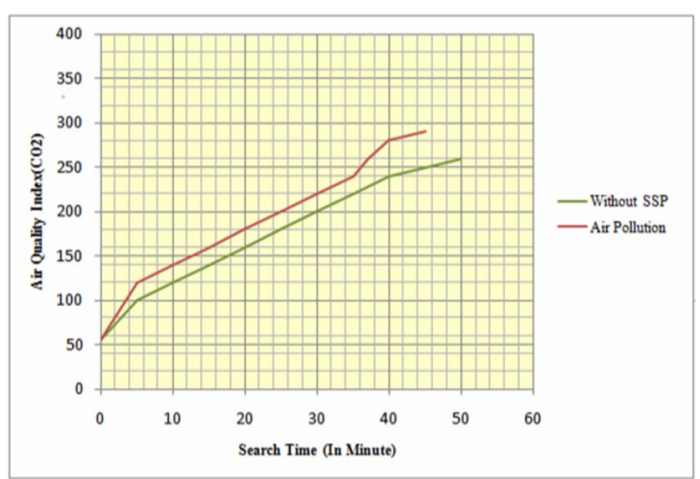

Fig. 5. Parking System without Shared Smart Parking

As shown in Fig. 6., the given graph depicts that with the help of a shared smart parking system, the searching time of the user for parking will be decreased, traffic will be reduced, and hence it will result in reducing air pollution.

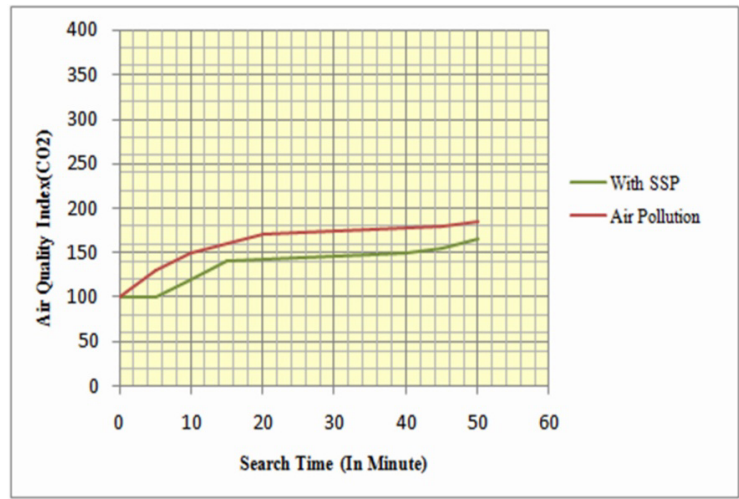

Fig. 6. Parking System with Shared Smart Parking

\section{Conclusion}

In this paper, a shared smart parking is proposed which facilitates the sharing of parking resources between the owners of parking slots and users. This system is not only helpful to the user to find the nearest suitable parking slots in minimum time but will also provide an effective solution to reduce air pollution and traffic problems.

\section{References}

1. T. N. Pham, M. F. Tsai, D. B. Nguyen, C. R. Dow, D. J. Deng, IEEE Access, 3, 1581-1591, (2015)

2. S. R. Basavaraju, International Journal of Scientific and Research Publications, 5, 12, 629-632, (2015)

3. B. M. K. Gandhi, M. K. Rao, Indian Journal of Science and Technology, 9, 17, 1-6, (2016)

4. C. Shiyao, W. Ming, L. Chen, R. Na, IEEE, 2014 Sixth International Conference on Measuring Technology and Mechatronics Automation ,741-744, (2014)

5. J. Xiao, Y. Lou, J. Frisby, TRANSPORT. RES. BMETH. , 112, 19-39,(2018)

6. W. Alajali, S. Wen, W. Zhou, Springer, International Conference on Security, Privacy and Anonymity in Computation, Communication and Storage,641-652,(2017)

7. E. Simhon, C. Liao, D. Starobinski, 2017 IEEE Conference on Computer Communications Workshops (INFOCOM WKSHPS) , 641-646, IEEE, (2017)

8. Y. Zheng, S. Rajasegarar, C. Leckie, Sensor Networks and Information Processing, (2015)

9. S. Yang, W. Ma, X. Pi, S. Qian, Transp. Res. Part C Emerg. Technol. , 107, 248-265, (2019)

10. J. Hu, D. He, Q. Zhao, K. K. R. Choo, IEEE CONSUM. ELECTR. M. , 8, 4, 45-49, (2019)

11. F. I. Shaikh, P. N. Jadhav, S. P. Bandarkar, O. P. Kulkarni, N. B. Shardoor, Int. J. Comput. Appl. , 140, 12, (2016) 
12.https://en.wikipedia.org/wiki/Ra

dio-frequency identification 